FORMATION Formation emploi

Revue française de sciences sociales

145 | Janvier-Mars 2019

Varia

\title{
Que signifie professionnaliser ? École de commerce versus école d'ingénieurs
}

What does it mean to professionalize? Business schools versus Engineering schools

Was bedeutet es, sich zu qualifizieren? Business School versus Ingenieurschule ¿Qué significa profesionalizar? Escuela de negocios versus escuela de ingeniería

\section{Francis Guérin et Hédia Zannad}

\section{OpenEdition}

Journals

Édition électronique

URL : https://journals.openedition.org/formationemploi/6865

DOI : 10.4000/formationemploi.6865

ISSN : 2107-0946

Éditeur

La Documentation française

Édition imprimée

Date de publication : 15 avril 2019

Pagination : 29-51

ISSN : 0759-6340

Référence électronique

Francis Guérin et Hédia Zannad, «Que signifie professionnaliser ? École de commerce versus école d'ingénieurs », Formation emploi [En ligne], 145 | Janvier-Mars 2019, mis en ligne le 01 janvier 2021, consulté le 03 septembre 2021. URL : http://journals.openedition.org/formationemploi/6865 ; DOI : https://doi.org/10.4000/formationemploi.6865

(c) Tous droits réservés 


\title{
Que signifie professionnaliser ? École de commerce versus école d'ingénieurs
}

\author{
FrANCIS GUÉRIN \\ Maître de Conférences à I'Institut national des sciences appliquées (Insa) Rouen Normandie et \\ chercheur titulaire au DySoLab (Laboratoire des dynamiques sociales) - EA 7476 - Université de \\ Rouen Normandie \\ HÉDIA ZANNAD \\ Professeure associée NEOMA Business School - Campus de Rouen
}

Résumé

Que signifie professionnaliser ? École de commerce versus école d'ingénieurs

En mobilisant la théorie de la cité par projets et l'économie des conventions, ainsi que deux études de cas, cet article montre comment une école d'ingénieurs et une école de commerce opèrent une " désingularisation » des relations des étudiants à leur environnement. Pour ce faire, ces écoles circonscrivent l'univers dans lequel la mise en forme à laquelle elles procèdent sera reconnaissable et valorisée, l'objectif étant de réduire les incertitudes quant à la capacité de ces étudiants à trouver un emploi et à leurs opportunités de carrière. L'article décrit une professionnalisation "intégrée ", s'appuyant sur la puissance du "projet " en école de commerce et une professionnalisation "scindée ", séparant nettement le scolaire et le non-scolaire, en école d'ingénieurs.

Mots clés : enseignement supérieur, grande école, professionnalisation, projet, économie des conventions, selection, méthode pédagogique, projet professionnel

Abstract

What does it mean to professionalize? Business schools versus Engineering schools

On the basis of different theoretical approaches such as the Projective City or the Convention school and two case studies, this paper shows how engineering and business schools shape their students' relationship to themselves and to the environment in order to reduce uncertainties concerning employment opportunities. Because the figure and the competencies of engineers and-all the more-managers are fuzzy, engineering and business schools exert efforts to "shape" them in a figure that can be recognized and valorized by private companies.

Keywords: higher education, elite school, professionalization, project, economy of conventions, selection, teaching method, career project

Journal of Economic Literature: I 23 ; J 24

Traduction : Auteur.e.s 


\section{Introduction}

Si l'école d'ingénieurs et l'école de commerce étudiées sont nourries par une même logique - la mise en adéquation entre formation et emploi, mais aussi, de façon croissante, entre personne et marché - le type de professionnalisation proposé diffère notablement.

L'article met en évidence ces similitudes et différences entre les dispositifs de préparation au marché de l'emploi à l'œuvre dans ces deux établissements d'enseignement supérieur. Il s'appuie sur une enquête qualitative menée auprès d'étudiants, enseignants et personnels administratifs d'une école d'ingénieurs (Institut national des sciences appliquées, ou Insa, Rouen Normandie) et d'une école de commerce (NEOMA Business School).

La théorie adéquationniste n'est pas mobilisée ici. Selon celle-ci, d'une part, la professionnalisation (doit) consiste(r) à améliorer l'adéquation entre emploi et formation ; d'autre part, l'insertion socioprofessionnelle (définie comme le passage réussi entre formation et vie active (Fournier \& Monette, 2000)) serait facilitée par l'acquisition de savoir-faire spécifiques à un métier ou à un champ professionnel dès la formation initiale (Charles, 2014).

$\mathrm{Au}$ contraire, nous avons adopté une approche inductive en partant du terrain. Et, de fait, dans aucune des deux institutions, nous n'avons observé cette logique de pré-professionnalisation typique du modèle adéquationniste, mais plutôt des professionnalisations respectivement "intégrée " (école de gestion) et "scindée " (école d'ingénieurs). En école de commerce, la professionnalisation est "intégrée " dans la mesure où l'école intervient, en son nom, sur la totalité de la personne - dans ses dimensions scolaire, affective et sociale - comme sur l'intégralité de sa carrière. En école d'ingénieurs, au contraire, la professionnalisation et le savoir académique constituent, sinon dans la réalité, du moins dans la perception des personnes interrogées, deux sphères séparées.

Dans un premier temps (1), nous spécifierons les deux terrains, ainsi que la méthodologie adoptée, pour l'enquête qualitative menée entre 2012 et 2015 auprès de 65 étudiants, enseignants et personnels administratifs des deux écoles étudiées. Dans un deuxième temps (2), nous caractériserons leurs modèles de professionnalisation et les "paradigmes " dans lesquels ils s'inscrivent : le " projet " dans l'école de commerce et la "projection " en école d'ingénieurs. Enfin (3), en partant de ces observations de terrain, nous mobiliserons les corpus théoriques pouvant le plus utilement les éclairer. Ainsi, la cité par projets (Boltanski, Chiapello, 1999) et l'économie des conventions (Thévenot, 1986 ; Gendron, 2006), bien que présents sous des formes et intensités différentes dans les deux institutions, nous ont aidés à expliquer les différences d'investissements de forme ${ }^{1}$ consentis par l'école de commerce et l'école d'ingénieurs étudiées pour assurer l'employabilité - ou aptitude à l'emploi (Finot, 2000) - de leurs diplômés.

1. On peut les définir de manière très générale comme des tentatives pour établir des formes d'équivalence (codes, standards, coutumes, etc.) dotées d'un pouvoir de coordination (ici, entre le monde des entreprises et celui de l'enseignement supérieur). 


\section{Présentation des terrains et méthodologie mobilisée}

Les deux écoles étudiées se distinguent tant par leur mode de sélection que par la nature et le poids de leur dispositif de professionnalisation, mais sont toutes deux relativement homogènes quant à leur statut d'écoles de « deuxième rang " et leur localisation en Normandie. Cela peut permettre d'établir des comparaisons entre elles, mais qui n'auront évidemment pas, comme toute étude de cas, de valeur généralisable à l'ensemble des grandes écoles. De même, la constitution de l'échantillon et la méthode de recueil et d'analyse des données ont été le plus similaires possible dans les deux organisations, afin que les perceptions et discours produits par les acteurs soient réellement commensurables les uns aux autres.

\subsection{Deux institutions d'enseignement supérieur aux profils différenciés}

Une présentation rapide des deux institutions permettra de les resituer à la fois dans leur logique de sélection et dans leur manière d'envisager la " professionnalisation " (nous définirons ce terme plus loin) de leurs étudiants.

1.1.1 La sélection : priorité à l'excellence scolaire ou à la personnalité des candidats?

NEOMA Business School fait partie des dix premières écoles de commerce françaises. Créée en 1871, elle compte plus de 57000 diplômés répartis en France et dans le monde. La sélection pour le cursus Grande École se fait après deux années de « Prépa », sur la base de l'excellence scolaire, mais également sur la personnalité. Ainsi, l'entretien pèsera in fine pour environ $25 \%$ de l'ensemble des notes du concours. Y seront abordées les questions récurrentes sur les traits de personnalité, motivations et (éventuel) projet professionnel du candidat.

L'Insa Rouen Normandie, fondé en 1917, s'inscrit a priori, par son pragmatisme affirmé et sa vocation résolument industrielle, parmi les écoles d'ingénieurs constituant un «contremodèle dominé » à l'excellence polytechnicienne (Bouffartigue, Gadéa, 1996, p. 7), même si notre enquête révèle une réalité plus ambiguë. L'école figure au cinquième rang des établissements proposant un cursus en cinq ans et est $24^{\text {ème }}$ sur les 174 écoles classées, en 2018, par le magazine L'Étudiant. Elle recrute les deux tiers de ses étudiants directement post-bac. La sélection s'y opère avant tout sur dossier et résultats scolaires (la quasi-totalité des admis ont une mention bien ou très bien au bac $S$ ), l'entretien ultérieur servant alors surtout à " filtrer » les profils inadéquats sur le plan psychologique et à « faire la promotion » de l'école auprès des candidats. 


\subsubsection{Des dispositifs pédagogiques et professionnels différenciés : classique versus expérientiel}

Parallèlement aux cours " classiques " offerts par les départements académiques, le centre de carrière de NEOMA (dénommé CareeR CenteR) propose aux étudiants des ateliers d'aide à la rédaction de $\mathrm{CV}$ et à la passation d'entretiens de recrutement. En outre, un séminaire de connaissance de soi et un module de construction du projet professionnel tous deux obligatoires et valant des crédits ECTS (European Credits Transfer System) visent à réduire leur indécision vocationnelle et à faciliter leur insertion sur le marché du travail $l^{2}$. Lors de la soutenance finale de ce projet, les membres du jury - académique et/ ou professionnel - doivent être convaincus de la cohérence entre les caractéristiques de l'étudiant (personnalité et aptitudes), ses expériences (associatives et stages) et son projet professionnel. La pièce maittresse de ce dispositif est le stage long en entreprise, mais les étudiants qui le souhaitent peuvent le remplacer par la prise de responsabilité dans une des nombreuses associations de l'école ${ }^{3}$. Ces associations, en particulier lorsqu'elles occasionnenent une campagne électorale (bureau des élèves - BDE, bureau des sports - BDS, junior entreprise - JE), donnent également l'opportunité d'appréhender les différentes facettes et réalités d'une élection, avec ses gagnants, ses perdants, ses coalitions et ses trahisons $^{4}$. Elles constituent, de ce fait, des interlocuteurs recevables face aux enseignants. Ainsi, même si l'investissement associatif est très chronophage et entre parfois en rivalité avec le rythme et la charge de travail scolaires, il est largement encouragé par l'ensemble de l'institution, enseignants compris, tant l'apprentissage de la réalité économique et l'acquisition de compétences managériales qu'il occasionne font consensus.

Par contraste, le dispositif de l'école d'ingénieurs est beaucoup plus léger. S'il existe des ateliers obligatoires de préparation des CV et lettres de motivation, la vie associative et expériences extra-académiques ne sont alors ni prises en compte dans la scolarité, ni intégrées dans une aide à la formalisation du projet professionnel, qui n'existe pas. Des « journées des métiers " (diplômés venant présenter leur activité), destinées aux étudiants des départements de spécialité, et une "Approche des métiers » visant les étudiants de premier cycle, toutes obligatoires, existent néanmoins pour informer les étudiants quant aux débouchés possibles. Quelques départements offrent la possibilité de réaliser des projets de longue durée autres que purement scientifiques ou menés dans un cadre certifiant (Iso 9001) pour un client industriel ou institutionnel. Les stages - exécution en fin de première

2. "Le Master Grande École de Rouen Business School a l'objectif de répondre aux exigences d'un marché de l'emploi qui sinternationalise et de faire de l'étudiant un véritable acteur de son savoir. Flexibilité du cursus, construction du projet professionnel, pédagogie de l'action, internationalisation des parcours... Autant de mesures visant à donner à nos diplômés les meilleures chances de succès" (extrait du site internet de l'école).

3. On comptait, en 2016, 80 associations et 500 événements, mobilisant 800 étudiants et 2,4 millions d'euros de budget annuel.

4. Pour reprendre l'expression du directeur d'HEC, Bernard Ramanantsoa, dans le documentaire de cinquante-cinq minutes de Christophe Lamotte sur HEC : HEC, derrière la porte étroite (1988). 
année, technique en fin de troisième ou quatrième année, ingénieur en fin de cinquième année - correspondent aux exigences de durée et de contenu édictées par la Commission des Titres d'Ingénieur (CTI). Depuis 1934, elle protège le titre d'ingénieur et "fonctionne de fait comme un dispositif de 'certification a priori' "(Bousquet, Grandgérard, 1996, p. 77).

\subsection{Méthodologie : une enquête qualitative longitudinale auprès des acteurs-clés des deux institutions}

Souhaitant avant tout faire "parler " le terrain plutôt que lui imposer un cadre explicatif prédéterminé, nous avons à la fois procédé par entretiens semi-directifs, en veillant à ce que les profils interrogés permettent une réelle comparaison entre les deux écoles, et adopté une approche inductive la plus ouverte possible.

\subsubsection{Interroger des (futurs) cadres au même moment de leur parcours}

Dans un premier temps, nous avons interrogé des étudiants de niveau $\mathrm{Bac}+4$ ou $\mathrm{Bac}+5$ (quatrième ou cinquième année dans l'école d'ingénieurs et deuxième année du programme grande école de la Business School), tous "statistiquement " appelés à devenir cadres. Étant en milieu ou en fin de parcours de formation, ils ont une bonne vision d'ensemble des moyens mobilisés par leur institution pour les aider à se professionnaliser.

Lâge des étudiants interrogés dans les deux institutions est donc sensiblement le même 22 ans en moyenne. Nous avons pris la précaution d'interroger la même proportion de garçons et de filles que celle existant dans les institutions observées (environ $50 \%$ - $50 \%$ en école de commerce et $30 \%$ de filles - $70 \%$ de garçons en école d'ingénieurs).

En revanche, la variable « origine sociale " n’a pas d'emblée été explorée dans cetté étude, qui mettait plus l'accent sur « l'offre » de formation et de professionnalisation (intentions, dispositifs, nature) que sur les caractéristiques sociales et personnelles de ses destinataires.

Dans un second temps, nous avons interrogé, deux ans après leur sortie, des diplômés issus des mêmes cohortes, afin d'étudier la manière dont ils s'approprient (ou non) ces intentions et outils, ainsi que leur perception de leur propre processus de professionnalisation. Le but était d'interviewer, d'une part, des étudiants ayant été confrontés à l'essentiel du processus ; d'autre part, des jeunes diplômés ayant un regard critique et ex-post quant à l'utilisation qu'ils avaient pu faire des instruments mis à leur disposition ou quant à leurs pertinence et utilité.

Enfin, plusieurs entretiens complémentaires ont été réalisés auprès d'enseignants et responsables de chaque école. Il s'agissait à la fois de mieux connaître les dispositifs de professionnalisation mis en œuvre et de recueillir leur perception quant aux résultats obtenus. 


\subsubsection{Analyse et recueil des données : une approche inductive}

À l'inverse de la démarche déductive, la démarche inductive ne vise pas l'accumulation de la connaissance, mais l'explication d'un problème dans son contexte et dans sa dynamique, de manière globale. Elle part d'observations sur quelques cas particuliers, et, sans pour autant nier la connaissance préalable et les hypothèses déjà acquises (Eisenhardt, 1989), mène à une hypothèse ou un modèle scientifique (Mouchot, 2003). Elle s'appuie sur différentes stratégies utilisant prioritairement la lecture détaillée des données brutes pour faire émerger des catégories et/ou un «sens " à partir des interprétations du chercheur qui mobilise ces données brutes.

Elle est susceptible de donner lieu à trois types de résultats ou généralisations : a) généralisation empirique directe, b) jugements de probabilité ou c) réforme des théories, hypothèses ou intuitions à la lumière de l'expérience et de son analyse. Ici, c'est à cette dernière acception de l'induction que nous nous référons : les objectifs de recherche, tels qu'ils sont formulés, fournissent un point de vue, une perspective au chercheur pour conduire l'analyse de ses données, mais ils ne constituent pas une série d'attentes à produire, c'est-à-dire des résultats spécifiques à obtenir "à tout prix ». Notre démarche vise à "l'exploration et l'approfondissement d'une singularité accessible à l'observation " (Passeron, Revel, 2005, p. 9). L'objectif est de pouvoir ensuite, le cas échéant et sans savoir a priori si cela s'avérerait possible ou pertinent, "en extraire une argumentation de portée plus générale, dont les conclusions seront réutilisables "(ibid.). In fine, l'analyse inductive n'est certes pas aussi "solide " que d'autres approches permettant le développement de modèles et de théories. Néanmoins, elle permet de fournir un ensemble de procédures simples et directes afin de produire de nouvelles connaissances liées aux objectifs de recherche initiaux du chercheur (Thomas, 2006).

En cohérence avec les exigences de l'approche inductive, nous avons réalisé, dans chaque institution, des entretiens semi-directifs fondés sur des guides d'entretien les plus communs possible entre les deux institutions, compte tenu des idiosyncrasies « locales».

Les thèmes principaux, assortis de questions de relance, en étaient, pour les étudiants : 1) les objectifs professionnels à l'issue de la formation ; 2) les moyens de préparation à leur futur rôle professionnel et la perception/évaluation portée sur eux ; 3) les changements et progressions que les dispositifs mis en œuvre ont ou non permis de développer chez les étudiants.

Pour les diplômés, le guide était axé sur le parcours professionnel et l'emploi actuel, d'une part, et sur la manière dont l'école avait ou non (contenus de formation, dispositifs de préparation au monde professionnel au sens large) favorisé ce parcours et l'obtention/occupation de l'emploi actuel, d'autre part.

Enfin, il nous faut insister sur le fait que le matériau recueilli au cours des entretiens consiste évidemment, pour l'essentiel, en des perceptions et des représentations. Notre appartenance aux institutions concernées nous a donné par ailleurs accès (observation 
participante) à la réalité des pratiques mises en œuvre par chacune d'elles et à leurs discours internes " officiels" en termes de professionnalisation. Pour autant, nous ne restituons pas ici ces données, mais les perceptions et les réactions, souvent très hétérogènes, voire critiques, au regard des intentions, des discours et des outils développés, que chaque étudiant ou diplômé s’appropriera de manière singulière.

\section{Deux « modèles » de professionnalisation paraissant très différenciés}

Précisons d'emblée que, dans tout ce qui suit, nous entendrons par professionnalisation ou acquisition d'une professionnalité (Béduwé, Mora, 2016, pp. 63-65) l'ensemble des dispositifs visant l'acquisition/transmission (quand c'est l'institution elle-même qui en prend en charge le développement) et/ou l'explicitation/formalisation de compétences de trois ordres, constitutives de la professionnalité :

- les compétences « disciplinaires " ou "spécifiques » liées à la spécialité ou à la filière, incluses dans un champ professionnel précis ou visant l'exercice d'un groupe de métiers bien identifié ;

- les compétences « d'acculturation au monde du travail » renvoyant à la connaissance du marché et du monde du travail, des codes et normes qui y prévalent, des manières de s'y comporter ou de s'y intégrer ;

- les compétences "distinctives ", enfin, qui permettent de se différencier au sein d'un marché de l'enseignement supérieur massifié autant que démocratisé.

Dans chacune des deux écoles domine un «paradigme » différent de la professionnalisation. L'école de commerce affirme et revendique explicitement l'importance de mettre toute expérience personnelle, scolaire, associative au service de l'élaboration d'un "projet ". L'école d'ingénieurs, elle, se contente d'une référence et d'une "projection" omniprésentes, mais moins élaborées et instrumentées, dans la figure de l’ingénieur.

\subsection{Le projet professionnel, mode d'intégration totale en business school}

Dans l'école de commerce, on observe un phénomène qui n’apparaît pas dans l'école d'ingénieurs : l'injonction adressée à tous les étudiants - et ce, dès le début de la formation, voire dès les jurys de pré-admission - à construire un projet professionnel au service duquel les activités, les temps, les relations et les expériences de l'individu devront se placer pour l'enrichir.

En effet, l'institution met tout en œuvre pour permettre aux étudiants de se construire, par les choix d'options, les stages, les sujets de projets, les années d'immersion en entreprise ou de substitution à l'étranger, un profil professionnel déterminé. C'est la visée de 
toutes les pratiques, qu'elles soient pédagogiques - comme les filières de spécialisation centrées sur des métiers - ou d'accompagnement, comme le Centre de carrières (CareeR CenteR), qui guide les étudiants en matière de connaissance de soi, de compréhension du marché du travail et de communication interpersonnelle. Au-delà du CareeR CenteR, focalisé sur le projet de carrière professionnelle, tout est prétexte à alimenter le projet de l'étudiant, y compris ses activités personnelles et associatives. Même un projet alternatif est un " projet », au sens où il crée une dynamique d'orientation de l'identité professionnelle dans une direction affirmée :

"Contrairement à ce qu'on trouve à l'université, le fait d'alterner des périodes académiques et des périodes professionnalisantes en école de commerce permet d'identifier leurs compétences. On les pousse à faire des liens entre leurs connaissances théoriques et la réalité de la vie professionnelle. » (chargée du développement du projet professionnel des étudiants)

"On essaie de développer l'esprit d'entrepreneur, de prendre des risques, de poursuivre inlassablement une occasion de développer un projet ou de créer une entreprise. " (enseignant, responsable de la majeure entrepreneuriat)

Ainsi, les expériences, stages et rencontres - esquissant déjà des réseaux utiles - et les activités personnelles sont une source d'affermissement d'un projet professionnel. Les discours des étudiants interrogés confirment le propos de Lazuech (2000) : lorsque la représentation de l'avenir est claire, les choix successifs opérés sont plus rationnels et s'ancrent plus fortement dans cet avenir probable. A contrario, si elle est incertaine, l'étudiant peine à faire des choix dans le présent :

"J'ai fait l'école intelligente, c'est-à-dire que j'ai pris tout le contenant et les outils qui nous sont donnés pour les alimenter. L'adéquation cours/profession est inatteignable, mais l'école ne peut pas obliger les étudiants à se servir des outils au service de leur carrière. Moi, je voulais l'Allemagne, je voulais travailler dans un gros groupe, mais ayant des ramifications avec la France et je voulais du B to B. Ceux qui ont bayé aux corneilles, ne sont pas partis à l'étranger, n'ont pas fait de réseau et enchaînent les CDD. Moi, j’avais déjà signé mon contrat avant de terminer mon cursus. " (diplômée)

"Je suis en contrat de professionnalisation en alternance avec l'école des Ponts et Chaussées (Mastère spécialisé en Supply Chain) dans une entreprise française, en banlieue parisienne, de fournisseurs d'ingrédients de boulangerie en B to B. Les cours m'intéressent beaucoup car ils sont très techniques, très stimulants, ce qui m'a manqué en école de commerce. Mais jaurais aimé travailler directement dans une grande entreprise d'agro (Nestlé, par exemple), mais mes stages étaient trop différents les uns des autres. Il n'y avait pas de fil conducteur dans ce que j'ai entrepris. À l'école, on n'a pas assez d'orientation, quand on ne sait pas ce qu'on veut, on est perdu. Je n'ai pas eu les moyens de mon indépendance par rapport à ma famille, du coup, car je ne savais pas quoi faire. " (diplômé)

Si le projet professionnel constitue la clé de voûte du système, c'est parce que l'institution fait le pari que l'investissement des étudiants dans le champ associatif est central 
dans l'acquisition des aptitudes et habitus managériaux. A contrario, la dimension proprement scolaire et académique, pourtant énormément développée ces dernières années, sous l'impulsion des standards et des certifications internationaux, reste, in fine, secondaire : l'idéal des recruteurs français est postulé et représentés-comme proche du portrait-robot du manager des manuels de GRH : dynamique, relationnel, responsable et engagé, plutôt que scolairement brillant (Lazuech, 1999).

Le but de l'école de commerce est donc d'offrir la plus large palette possible de dispositifs expérientiels et d'accompagner les choix des étudiants. Pour ce faire, le projet reste le plus sûr moyen de canaliser les énergies dans la direction souhaitée. En effet, un étudiant - même brillant sur le plan scolaire - qui ne construit aucun projet professionnel, ne s'investit dans aucune association et erre de stage de marketing en stage humanitaire, constitue le profil le plus difficile à gérer pour la Direction comme pour les enseignants de l'école. Sans inculcation des codes de conduite du manager - qu'apporte pour l'essentiel l'investissement associatif - et sans projet professionnel orientant leur comportement, la formation des managers est vouée à l'échec, ne serait-ce que parce que les débouchés " naturels " des diplômés (grandes entreprises et administrations françaises) ne vont plus de soi et que le modèle même des grandes écoles est chahuté par les évolutions internationales (Fournas (de) \& al., 2008).

En effet, l'éventail des choix professionnels possibles s'est fortement diversifié et le choix via un projet devient à la fois une possibilité et une nécessité qui ne s'imposaient pas avec autant de force dans le passé. Le " projet » est considéré comme la condition sine qua non pour qu'un étudiant - en particulier s'il est arrivé en école de commerce non par vocation mais pour son excellent niveau scolaire -, puisse acquérir les dispositions techniques et comportementales requises par le rôle professionnel qui l'attend :

"On essaie de développer, chez les étudiants, la curiosité, l'ouverture d'esprit, le sens des responsabilités et l'engagement dans la durée, vis-à-vis de leur entreprise et de leur équipe. Ils doivent pouvoir mener un projet du début à la fin. Toute cette expérience professionnalisante fait une grosse différence avec l'université, car elle leur permet d'identifier leurs compétences, leurs zones de confort, de construire leur projet professionnel au fil du temps et de le tester. " (Responsable chargée du développement du projet professionnel des étudiants)

Dès lors, le scolaire ne constitue qu'une des composantes d'une formation "intégrée " qui touche tout ce qui peut être travaillé au-delà du cadre scolaire stricto sensu, y compris l'apparence physique et toutes les manifestations extérieures de l'aisance. Les étudiants sont certes préparés à la vie professionnelle, mais aussi à la vie tout court.

5. Si l'école subit effectivement des influences extérieures modelant le curriculum proposé aux étudiants, nous pensons, à l'instar de Lemaître (2009), que le discours pédagogique de l'école de commerce constitue une "recontextualisation" de ces demandes, c'est-à-dire une "mise en représentaion des savoirs, orientée vers des finalités éducatives, objet d'une médiation interne " (ibid, p. 19), grâce à laquelle l'institution sélectionne, organise et désigne les savoirs et activités légitimes. 
Une grande valeur est accordée aux activités d'expression de soi (art, sport, etc.) ou orientées vers le relationnel (humanitaire, caritatif, etc). L'éloignement de l'école par rapport au centre-ville, le déracinement géographique, la coupure opérée avec les anciens camarades par la Prépa. et la décohabitation avec les parents favorisent le recentrage sur les activités associatives. Les candidats en entendent parler par les " admisseurs " - les étudiants en charge de l'accueil des admissibles - dès les jurys d'oraux.

L'homogénéisation sociale et l'acculturation managériale qui en découlent sont d'autant plus efficaces qu'elles s'opèrent de façon discrète, voire souterraine, puisqu'elles s'appuient sur l'adhésion librement choisie d'étudiants qui deviennent les vecteurs de leur propre apprentissage :

"Les cours m’ont peu servi, mais le côté associatif, oui, car j’avais organisé un gros concours d'équitation qui m'a appris à mener un projet jusqu'au bout (800 cavaliers sur trois jours) : j'ai appris à gérer un budget de $85000 €$ et mon stress. À chaque fois que je me fais engueuler ou que je fais une erreur dans mon job, janalyse calmement la situation et me dis que j'ai déjà géré un projet de bout en bout. " (étudiant)

"Grâce à mon parcours en école de commerce, je suis à près de trois ans de stage; je ne changerais ma formation pour rien au monde. Je savais comment fonctionnaient les stages et les Forums Entreprises car j'en avais organisé un. Donc, j'ai choisi Mazars car le stage durait quatre mois. À l'issue de ma formation, j'avais deux propositions d'emploi : ERDF et Canal +, j’ai choisi le premier. " (diplômé)

La logique projet intègre donc progressivement le scolaire - discours pédagogique vertical - au professionnel - discours pédagogique horizontal. Elle fait du projet un " tout » transcendant le temps (possibilité d'adhérer à vie à l'association des diplômés, temps hors école valorisés sur le CV, modelage du présent en fonction de l'avenir projeté) et l'espace ("tribus » de diplômés dans toutes les régions de France, mais aussi du monde) et propose la figure d'un individu qui confond le professionnel et la personne.

Pour autant, les entretiens révèlent des réactions très diverses de la part des étudiants face à cette ambition. À côté des "managers en herbe " qui y souscrivent dès le départ et agissent en "professionnels » en saturant leur agenda d'expériences "valorisables » dans un futur déjà identifié, nous avons rencontré des " réfractaires ». Ils restent critiques visà-vis des normes dominantes et des comportements attendus. Cela les incite, soit à aller chercher hors de l'école (par exemple dans des cursus à l'étranger) l'exigence intellectuelle que l'école ne leur offre plus, soit à se désinvestir totalement de la vie de l'école. Mais les discours, l'organisation de la scolarité, l'offre pédagogique et l'investissement extra-scolaire finissent aussi par orienter et acculturer assez sûrement des " co-équipiers " (qui jouent le jeu au départ par esprit collectif) ou des « investis » (qui cherchaient initialement à faire vivre leurs passions personnelles, mais finissent par adopter un projet professionnel " conforme ») - y compris ceux qui en sont éloignés au départ - vers l'univers de destination managérial. 


\subsection{La figure de l'ingénieur : une projection multiforme plutôt qu'un projet directeur}

Dans l'école d'ingénieurs règne un paradigme moins prescriptif : la " projection » dans la figure de l'ingénieur. Cette figure, particulièrement éclatée et multiforme, est sans cesse invoquée comme horizon certes indéfini, mais néanmoins certain, tant par les étudiants que par les responsables de l'institution. Ces derniers admettent que l'école et surtout ses enseignants sont avant tout soucieux d'excellence scientifique dans leur propre discipline et ne se préoccupent guère de préparer les étudiants à un avenir professionnel qu'ils leur laissent le soin d'identifier eux-mêmes.

À un premier niveau d'analyse, on peut le voir comme une conséquence de l'académisation progressive qu'ont connue la plupart des écoles d'ingénieurs ${ }^{6}$, à l'exception de celles qui, comme Polytechnique, se situaient d'emblée dans le modèle de l'excellence et ont été très tôt marquées par un modèle très académique et une distance avec le monde industriel (Shinn, 1978). Dès lors, nombre d'écoles d'ingénieurs, comme l'Insa dans les années 1990 et 2000, ont progressivement substitué des enseignants-chercheurs aux enseignants praticiens chargés d'apporter aux étudiants des connaissances et des savoir-faire d'ordre technique (Sonntag, 2007 ; Lemaître, 2011) :

"Aujourd'hui, le produit de sortie reste inchangé, même si, il faut l'admettre, l'école était très académique à l'origine, avec des enseignants-chercheurs qui tendent à vouloir produire des chercheurs. [...] Les professeurs d'université sont responsables de leur pédagogie, mais vont-ils bien vers l'entreprise? Il y a des cours magistraux, mais des pratiques comme le travail en ateliers sur des cas concrets, les jeux de rôles, etc. devraient être plus développées. [...] On part de loin dans ce domaine et pendant longtemps, nos formations ont été conçues comme des super masters. " (Directeur des formations, maître de conférences en énergétique)

"Le modèle des profs est certainement aussi une explication possible au comportement de nos diplômés: ils n'ont jamais mis les pieds en entreprise alors qu'ils en parlent sans cesse. Ils nont pas de culture de l'entreprise. Ce sont de bons enseignants-chercheurs, mais ils n'ont pas de vision du monde de l'industrie et de l'économie qui sont pourtant, de manière naturelle, le débouché de nos diplômés. Ils ont donc du mal à transmettre une connaissance qu'ils n'ont pas à leurs étudiants ou à leur dire quels réflexes il est important d'avoir dans ce monde. " (Directrice de la communication)

La progressive académisation de l'Insa, école spécialisée devenue généraliste (voir Hatchuel, 2006, à propos de l'École des Mines de Paris et son parcours comparable), en fait un " prétendant » qui affirme ses ambitions et prétentions en se conformant à ce qui a longtemps

6. Mais aussi, avant qu'elles ne se tournent vers le modèle des business schools américaines, certaines écoles de commerce, puisqu' "en conduisant les transformations d'HEC, Guy Lhérault [son directeur de 1958 à 1969] disait de manière presque obsessionnelle vouloir rattraper l'Ecole Polytechnique " (Fournas (de) \& al., op. cit., p. 34). 
été la marque des écoles dominantes. Cependant, elle crée une partition autrefois inexistante entre deux univers : historiquement, ses enseignants étaient bien plus proches de l'industrie et de la pratique.

Les étudiants eux-mêmes développent plusieurs arguments pour expliquer comment et pourquoi leur projection dans leur avenir professionnel est rarement précise et pourquoi leur présent n'est pas entièrement voué à préparer cet avenir.

Le principal est que, pour le meilleur ou pour le pire, l'ingénieur constitue un contenant aux contenus possibles fort larges et diversifiés.

Pour le meilleur, car " on trouve des ingénieurs dans à peu près tous les postes et dans toutes les fonctions de l'entreprise et on pourrait dire que ce mot ne veut à peu près rien dire. [...] ce qui caractérise les ingénieurs, c'est justement l'éventail des possibilités qui leur sont offertes après " (étudiant de cinquième année). Ces étudiants-là ont compris que, a fortiori dans une école qui se veut et se dit généraliste, l'ingénieur déborde depuis longtemps les frontières de l'industrie et de la technique. Ils en retirent une forte confiance en leur propre avenir, qu'il n’est donc pas nécessaire de surdéterminer.

Pour le pire, car cette extrême diversité rend difficiles les choix professionnels et ouvre tant de possibilités que certains se sentent écrasés et dépassés par la peur de se tromper dans leurs décisions.

Les uns estiment que la formation ouvre de nombreuses portes, mais craignent que certaines ne débouchent sur des impasses ou des passages étroits vers le marché du travail. Les difficultés liées au marché du travail - dont les ingénieurs ne seraient selon eux pas exempts - les conduisent parfois à affirmer, lors des entretiens et de manière très pragmatique, ne pas être trop exigeants et prêts accepter le poste qu'ils trouveront à la sortie. Néanmoins, si les marges de manœuvre leur paraissent réduites, ils estiment que le diplôme leur permettra d'en trouver un et aucun des interviewés n'a exprimé de doute à ce propos :

"Le marché du travail, ça reste quelque chose de vague pour moi. Je pense qu'on n'a pas la vision de plus loin. On ne se projette pas dans l'avenir. Je compte sur la qualité de mon stage ingénieur pour maider à trouver du travail, mais j'ai aussi peur de faire les mauvais choix, de menfermer dans un secteur, dans un domaine, où il n'y a pas de boulot. " [...] Ce qui me rassure un peu, ce sont les chiffres de placement publiés par le département. Ça montre que les diplômés se placent bien quand même et qu'ils trouvent du boulot, enfin, qu'il n'y a pas de raisons de trop s'inquiéter. " (étudiante de cinquième année)

Les autres pensent, quant à eux, que l'étiquette " ingénieur " qui sera accolée à leur titre, au terme de leur formation, est assez lisible et reconnue pour éviter de réfléchir davantage à leur avenir professionnel :

"À vrai dire, mon projet professionnel à court terme, c'est déjà de trouver du travail, tout simplement. Je suis débutant et je ne vais pas avoir de prétentions. Je prendrai ce qui vient et ce que je trouve, pourvu que ça rentre dans les objectifs de fond de l'institution et que ce ne soit pas 
trop à côté de la plaque par rapport à ce qu'on est supposé faire à la sortie d'une école d'ingé." . (étudiant de cinquième année)

Mais pour tous, " pessimistes " comme " optimistes ", préparer l'avenir en forgeant un projet élaboré et précis est contre-productif : d'une part, l'avenir est de plus en plus incertain et appelé à évoluer ; d'autre part, et surtout, l'école les prépare finalement assez bien à affronter un tel avenir, même si ce n'est pas de la manière attendue :

- si nombre de savoirs scientifiques et techniques ne serviront jamais et sont vite oubliés, les méthodes de travail que leur étude suppose sont, quant à elles, définitivement acquises et transposables à n'importe quelle situation ;

- le caractère généraliste de la formation, le fait même de survoler de nombreuses thématiques, de "sauter du coq à l'âne très vite et très souvent", sont autant de manières indirectes d'apprendre à s'adapter rapidement et à travailler sur des tâches et sujets très éclatés ;

- la pédagogie par projets, très répandue, mais aussi les travaux pratiques les obligent à devenir autonomes, à trouver eux-mêmes les solutions à des situations inédites et souvent très spécifiques qui ne font pas à proprement parler partie de leur cursus de formation.

L'autre argument des étudiants pour justifier leur faible degré de projection dans l'avenir est le besoin de se construire au présent, de travailler à leur développement personnel ou de rééquilibrer leur profil. Et l'école, par les espaces et les temps qu'elle laisse libres, leur offre un cadre propice à ce travail, qu'ils ne relient pour autant ni à une perspective strictement professionnelle, ni à l'optimisation de leur employabilité, mais bel et bien à eux-mêmes : les vides, les vacuités que laisse l'école - énorme différence avec l'école de gestion - sont avant tout dédiés à leur épanouissement et leur développement personnels. L'école leur apporte autant par les latitudes et marges qu'elle leur laisse que par ses exigences vis-à-vis d'eux :

"Pour moi, ce neétait pas gagné, à mon entrée à l'Insa, car je n'étais pas la plus jolie, ni la plus mince. Aujourd'hui, je cours tous les matins et je sais que je peux avoir confiance en mon corps, qu'il ne me lâchera pas. Pour acquérir cette confiance sur différents plans, il faut se connaître soi-même et ça, ça ne sapprend qu'en vivant des expériences qui permettent de se confronter à soi-même. Le rôle de l'Insa, dans tout ça, dans ce processus de maturation, n'a pas été joué par les études elles-mêmes, mais uniquement à travers le cadre que l'école offre. [... ] L'Insa donne à chacun la possibilité de devenir qui l'on veut être. "(étudiante de cinquième année)

"Le fait d'avoir les jeudis après-midi libres pour faire du sport, avoir des loisirs, voir ses amis ou avoir une vie sociale est quelque chose qui est très apprécié par les étudiants. Ça permet de faire autre chose que les cours, de s'aérer la tête et d'avoir une coupure dans la semaine pour penser à autre chose. [...] Moi, à titre personnel, à l'Insa, je me suis plus investi dans certains loisirs, comme la flûte, la chorale, le sport, que dans mes cours. " (diplômé, promotion 2015)

Ces activités extérieures sont quasi ignorées par l'organisation, qui ne les encourage, ni ne les valorise en termes de professionnalisation. Et les étudiants ne les relient dès lors 
ni à leur scolarité, ni à une quelconque forme de professionnalisation. Développées dans les marges de l'institution, elles sont vécues comme telles par les étudiants. Ainsi, l'école en est le cadre possible, mais n'est pas identifiée par eux comme en étant l'initiatrice ou l'organisatrice. Elles appartiennent en propre aux étudiants qui ne les réfèrent qu'au présent et à eux-mêmes, sans souci de valorisation actuelle ou future, d'intégration à leur curriculum ou de développement d'un "habitus " reconnu et admis par des employeurs potentiels. En contrepartie, les élèves de l'Insa expriment le besoin d'affirmer et cultiver ces goûts personnels qui ont été réfrénés, autant que de développer et laisser s'épanouir d'autres qualités que celles - scolaires - qui leur ont permis de réussir, mais ont nié les dimensions de leur personnalité avec lesquelles ils aspirent désormais à renouer.

Enfin, l'acquisition d'une professionnalité (Béduwé, Mora, op. cit.) est elle aussi "schizée » : la transmission de compétences " disciplinaires » liées à la spécialité est strictement dévolue aux enseignants-chercheurs dans le cadre des enseignements formels qu'ils délivrent sous forme de cours magistraux, travaux dirigés ou travaux pratiques. Mais les compétences « d'acculturation au monde du travail » et les compétences " distinctives » se forgent ici en dehors de ce cadre formel. Elles échappent aussi largement au contrôle de l'institution qui ne suscite même pas leur explicitation : visites d'entreprise, stages, interventions de professionnels, projets réalisés pour le compte d'un "client » extérieur, forums ou journées de rencontre avec les diplômés, etc. Ces formes de professionnalisation s'acquièrent donc surtout hors des temps, voire des lieux, administrés par l'école, laquelle se contente d'en tirer parti :

"L'école investit, même si c'est depuis peu, sur les associations, en essayant de leur donner une valorisation dans le cursus sous forme de crédits : ça a demandé trois ans pour arracher ça en comité des formations. De même, certains départements, à travers les projets personnels, encouragent les idées personnelles des étudiants et jouent le jeu. [...] En fait, on ne sait pas bien faire grandir nos étudiants et la conclusion qu'on peut en tirer, c'est que l'aspect formatif est mieux assuré que l'aspect comportemental. " (Directeur des formations et maître de conférences en énergétique)

Loin d'une institution qui tenterait d'intégrer totalement présent et futur, personnalité et professionnalité, scolaire et hors scolaire, ce modèle relève plus de la juxtaposition, de la séparation, voire de la schizophrénie, des temps, des expériences et des lieux de vie. 


\section{Quels cadres socio-économiques pour interpréter ces modèles?}

Nous pourrions en rester là et conclure sur des différences sensibles entre les dispositifs de professionnalisation des deux institutions, NEOMA se rapprochant effectivement plus de l'idéal de l'" intégration totale de l'individu "à l'école (Charles, 2015, p. 102), en général attribué aux grandes écoles (Bourdieu, 1989) ou d'une "pédagogie du savoir-être " (Lazuech, 1999).

Néanmoins, il est possible et nécessaire d'aller plus loin et de montrer que, apparemment différents, les comportements des deux institutions relèvent en réalité d'une même logique, d'une seule et même " économie » vis-à-vis de laquelle leurs positions et atouts diffèrent.

Deux cadres théoriques nourrissent ainsi l'analyse du matériau recueilli. D’une part, les investissements de forme (Thévenot, op. cit.) que doivent consentir non seulement une institution d'enseignement supérieur, mais aussi ses diplômés, pour réduire l'incertitude quant à la valeur de ces derniers ; d'autre part, la nature et le degré de professionnalisation développé qui prennent la forme, en école de commerce, de la cité par projets (Boltanski \& Chiapello, op. cit.).

\subsection{Investissements de forme et réduction d'incertitudes multiples}

Le concept d'investissement de forme, proposé en 1986 par Laurent Thévenot, s'inscrit dans l'économie des conventions. Il vise à dépasser l'analyse néo-classique, en particulier lorsqu'il s'agit de rendre compte de la relation d'emploi entre salarié et employeur, mais aussi de la formation d'un prix (ici, le salaire) qui ne se verra justifié qu'a posteriori. Pourtant, dans l'économie " réelle ", le montant du salaire est fixé a priori, alors que sa valeur économique réelle ne se révèlera qu'a posteriori. Cette capacité de régulation s'explique par l'existence d'autres formes de coordination non marchandes (la confiance, la réputation ou encore l'intérêt général) qui sont autant de conventions, parmi lesquelles figurent les « investissements de forme ».

Comment une entreprise voulant recruter un diplômé peut-elle réduire l'incertitude qui entoure sa valeur réelle ou son utilité potentielle ? En s'appuyant sur ce que Bénédicte Gendron (op. cit., p. 7) appelle des "indicateurs partiels ", c'est-à-dire des signaux, parmi lesquels figure le diplôme qui va "mettre en balance un coût et la généralité d'une forme qui sert d'instrument d'équivalence [ici, entre le salaire versé et les compétences qui le justifient] et qui est caractérisée par sa stabilité et son domaine de validité " (Thévenot, op. cit., p. 30). Plus la forme d'investissement sera durable (stabilité), générale (domaine de validité et de reconnaissance) et objectivée (dans un « outillage » technologique, juridique, scientifique, procédural, instrumenté), plus l'investissement s'avérera " rentable ». 
Enfin, Laurent Thévenot distingue deux types de formes : générales et spécifiques. Les formes d'État font partie des formes générales, a priori les plus robustes : l'engagement de l'État leur confère une généralité, une stabilité et, le plus souvent, un degré d'objectivation que n'auront pas d'autres diplômes ou titres reconnus plus localement, moins stables ou objectivés : "Les titres scolaires produits par l'État permettent donc une équivalence nationale et par conséquent un traitement indépendant du porteur particulier du fait de l'institution de la durabilité, du domaine de validité et de l'objectivité du titre" (Gendron, op. cit., p. 11). Ils renvoient un signal plus "fort » aux entreprises qui économiseront ainsi des coûts significatifs liés à l'opération de qualification des individus.

Le titre d'ingénieur délivré par une école publique comme l'Insa fait partie de ces formes d'État, et l'institution communique explicitement sur ce caractère d'établissement public. Si ce statut offre de faibles coûts d'inscription, il " désingularise " aussi les diplômés. Il réduit en effet significativement les inconnues que sont leurs qualités et compétences pour les firmes. De même, l'insistance sur le titre d'ingénieur, sa délivrance par le ministère de l'Enseignement supérieur, son caractère protégé et suspendu à l'accréditation des établissements et à l'habilitation de chaque spécialité par la Commission des Titres d'Ingénieur tirent le titre vers une forme d'État et rassurent les étudiants quant à leur valeur sur le marché du travail, sans qu'ils aient à produire des signaux individuels forts. La projection dans la figure de l'ingénieur paraît " suffisante » et assez protectrice pour que le temps et le lieu de l'école ne soient pas systématiquement - comme dans l'école de commerce référés à l'avenir professionnel dont la construction pèse davantage sur l'individu.

À NEOMA, le diplôme et surtout le travail associé de désingularisation détachent l'individu de sa singularité, de ses centres d'intérêt, de ses caractéristiques personnelles, au profit d'une objectivation valable sur le marché du travail et " équipée » de tout ce qui est mis en place par l'institution pour écarter des singularités relevant certes du savoir scolaire antérieur, mais aussi du savoir-être. Ainsi, toute expérience vécue est supposée être réinterprétée en termes de compétences acquises (c'est notamment à cette traduction qu'aide le CareeR CenteR). Ce propos rejoint les analyses de Marianne Blanchard (2015) qui montre que le succès de nombre d'écoles supérieures de commerce au «statut originel incertain » et à la "faible légitimité" ne doit pas tant à leur capacité à répondre à la double demande des entreprises friandes de diplômés «employables» et des étudiants ou de leur famille en quête de filières distinctives, qu'à un "travail actif de présentation de soi " qui leur permettra de devenir " grandes» en imitant d'autres établissements déjà reconnus (notamment les écoles d'ingénieurs et leurs prépas en deux ans).

Dans les formes d'État, l'équivalence a une telle force que la forme n'a pas besoin d'être aussi " outillée " que dans une forme intermédiaire, moins durable et objective, et d'un moindre domaine de validité. Ainsi, la moindre stabilité de la forme assurée par l'école de gestion la contraint à la retravailler sans cesse au cours du temps. Elle est notamment réaffirmée via un réseau de diplômés (publications, réunions, services offerts tant aux individus qu'à la " communauté ») auquel il est possible d'adhérer à vie et qui se constitue en 
«tribus» (sic) régionales (pour la France) ou nationales (pour l'étranger) continuant et maintenant le travail de mise en forme engagé durant la scolarité.

Mais l'affaiblissement relatif de la forme d'État offerte par le diplôme d'ingénieur explique aussi que l'Insa crée désormais des « interstices " permettant la mise en forme par d'autres : l'école ne sait pas instrumenter, outiller, une mise en forme autre que scientifique, tout en sachant que celle-ci ne suffit désormais plus pour des métiers d'ingénieurs de plus en plus écartelés entre éthos technique - celui du spécialiste, modèle traditionnel de l'ingénieur et éthos communicationnel - celui du manager, de plus en plus présent dans les métiers exercés ou visés par ses diplômés (Lemaître, 2011).

D'ailleurs, le caractère « scindé » de la professionnalisation assurée par l'Insa ou la moindre mise en forme effectuée par l'école d'ingénieurs, mais aussi les faibles investissements consentis dans ce domaine par les élèves ingénieurs, témoignent, selon nous, d'une institution et d'élèves qui ne se perçoivent pas comme étant exposés au déclassement relatif de la forme d'État que constitue le diplôme délivré. Ainsi, contrairement aux écoles de tout premier rang, l'Insa n'alimente que très peu la haute fonction publique et les états-majors d'entreprises et a donc été moins exposé à la concurrence accrue des formations de gestion ou étrangères (Atlan \& Grelon, 2009). L'implication croissante des entreprises dans le dispositif de professionnalisation, l'absence de contrôle par l'école sur cette implication ou ses effets - quels qu'ils soient - sur les étudiants « tirent » la forme mise en ouvre vers l'univers des formes dites « intermédiaires » : l'apport d'un complément « local » d'investissement de la part des entreprises est nécessaire pour que l'école continue à jouer son rôle. La scission identifiée lors des entretiens témoigne ainsi d'un glissement d'une forme vers une autre, dont les acteurs concernés (enseignants-chercheurs, étudiants, responsables de l'institution) n'ont pas pris la pleine mesure.

Les diplômés de l'école de commerce exposent d'autant moins les entreprises à la surprise et à la déception que l'école s'emploie à " mettre en forme " l'intégralité de leur personne et à la mettre au service d'un projet. Mais leur consentement à cette mise en forme présente pour eux à la fois des bénéfices et des coûts. En effet, elle concourt à réduire et à circonscrire les univers dans lesquels leur valeur sera reconnaissable et passible d'une mise en équivalence : pour l'essentiel, les grandes entreprises privées, quelle que soit leur nationalité. L'uniformisation de leur relation aux entreprises oriente de manière " naturelle » les étudiants et diplômés vers ces organisations dans la recherche de cette forme d'équivalence, en excluant d'autres horizons.

De fait, nos entretiens révèlent que les étudiants (ou les diplômés) interrogés ont peu à peu abandonné les stratégies de positionnement alternatives (monde associatif, PME petites et moyennes entreprises) qu'ils ont pu nourrir à un moment de leur scolarité. Dès lors, ils s'orientent vers les grandes entreprises, conformément aux attentes implicites de leur école de commerce et à leur univers de destination. Accepter cette mise en forme proposée par l'institution leur épargne aussi les affres de choix trop ouverts et apporte une certaine sécurité dans la recherche d'emploi ou la progression de carrière. 
En témoignent les étudiants qui, continuant à jouer la seule carte de l'excellence scolaire, se sont rendus "singuliers " par rapport à leurs pairs et ne se sont pas inscrits dans le même régime d'équivalence.

\subsection{Professionnalisation intégrée et « cité par projets »}

L'école de commerce consent de plus lourds investissements de forme que l'école d'ingénieurs dans la réduction des incertitudes liées à la valeur de son diplôme et à la figure du manager, moins reconnue et légitime, mais aussi plus récente, que celle de l'ingénieur. Mais quels traits revêt cette forme reconnaissable entre toutes et employable par l'entreprise?

Sans doute ceux de l' "entrepreneur ", dont l'activisme lui permet de réaliser son potentiel dans toutes ses conduites et toutes les sphères de son existence (Burchell, 1993), voire de se constituer lui-même en entreprise à faire fructifier (Rose, 1992). Ceux d'un individu qui, pris dans une succession de projets, étend ses réseaux, multiplie ses connexions et fait proliférer ses liens. "Le projet est précisément un amas de connexions actives propre à faire naître des formes, c'est-à-dire à faire exister des objets et des sujets, en stabilisant et en rendant irréversibles des liens. " (Boltanski \& Chiapello, op. cit., p. 157)

De fait, l'école de commerce s'inscrit dans une approche dynamique centrée sur l'individu et sa trajectoire tout au long de son cycle de vie (Gautié, 2003). Cela traduit un souci d'employabilité, entendu comme la préoccupation constante de développer des compétences pour l'insertion dans le marché du travail, voire, en termes managériaux, de se "rendre désirable " pour prendre part à des activités marchandes ou non marchandes (Périlleux, 2005).

L'école s'appuie sur un socle idéologique décrit par Boltanski \& Chiapello (op. cit.), Finot $(o p$. cit.) et Périlleux $(o p$. cit.), qui tient dans les thèmes clés de la " cité par projets " : la mobilisation de soi, la vie elle-même conçue comme une succession de projets, l'intensification de l'activité et la nécessité de penser compétence en référence constante au marché. Dans ce monde en réseau, où la mobilité discrimine les salariés, savoir " rebondir » de projet en projet en capitalisant des compétences transférables et valorisables sur le marché devient décisif.

Le rapport au temps, marqué par la saturation, d'une part, et par l'optimisation, d'autre part, préfigure le "vrai » manager ou cadre d'entreprise, nécessairement sur-occupé et dont le présent est "envahi " par des conduites intentionnelles qui orientent l'action et donnent sens à l'avenir désiré (Boutinet, 1990). Pour rendre ses compétences personnelles et professionnelles transférables d'une firme à l'autre, le sujet "employable » ménage son patrimoine - finances, santé, réseau relationnel, aptitudes cognitives et comportementales, ressources affectives et émotionnelles - dans toutes les arènes de sa vie, ce que Gordon (1991, p. 44) nomme la "managérialisation » de l'identité. 
Ce paradigme du projet introduit une homologie constitutive entre entreprises et individus qu'elles recrutent : gestion des capitaux (matériels et immatériels, culturels, sociaux et économiques), optimisation des ressources, mobilisation des énergies dans une direction donnée, etc. Les investissements de forme consentis par l'école de commerce et ses diplômés, qui sont à la fois "élevés " en termes d'effort et orientés vers l'idéologie managériale dominante en termes de contenu, poussent les étudiants qui " jouent le jeu » à devenir progressivement à la fois eux-mêmes des entreprises et entrepreneurs d'eux-mêmes.

Par différence, l'école d'ingénieurs continue de se référer à la plus classique " cité industrielle» (Boltanski \& Thévenot, 1991). L'efficacité et la science y constituent les valeurs de référence et les sujets valorisés sont l'expert, le professionnel, l'opérateur, porteurs de qualités telles que la fiabilité, la fonctionnalité et la validité scientifique. Si elle s'incarne dans (et se suffit de) la figure de l'ingénieur qui la spécifie, elle est aussi moins exigeante en termes d'investissement de forme. En effet, contrairement à la cité par projets, elle ne concerne que la sphère de la cognition et de l'agir, mais en aucun cas l'être tout entier dans ses caractéristiques personnelles et ses qualités intimes. La personne continue d'exister à côté du professionnel, sans s'y fondre ou s'y faire absorber. De même, la "scission " constatée au sein de l'école d'ingénieurs est dès lors moins étonnante, la question étant de savoir si la "grandeur » de cette cité reste(-ra) un mécanisme de coordination ou d'accord fort au sein des organisations.

In fine, c'est l'ensemble du curriculum - décorum institutionnel, contenus d'enseignement et parcours de formation - voire la conception de la profession de cadre, de ses compétences et de son rapport au monde que doit construire l'étudiant dans chacune des deux écoles, qui diffèrent (Lemaître, 2009). L'équilibre entre discours pédagogique vertical (Bernstein, 2007) - enseignements orientés vers des savoirs - et discours horizontal - savoirs professionnalisants et pédagogie active orientée problèmes et projets - $y$ est très différent (Lemaître, 2009). Si l'école d'ingénieurs continue de porter un discours pédagogique vertical et disciplinaire et délègue le discours horizontal à des porte-parole souvent externes, l'école de gestion, elle, surinvestit ce champ du discours pédagogique horizontal qui correspond bien plus aux qualités et compétences qu'elle s'attache à développer chez ses étudiants.

\section{Conclusion}

Confirmant le caractère multiforme que la professionnalisation peut revêtir (Lemaître, 2011), notre recherche révèle deux archétypes de professionnalisation très différents en intention, en nature et en portée, renvoyant à deux formes d'injonctions implicites : "deviens qui tu es " pour l'école de commerce, "deviens ce que tu veux " pour l'école d'ingénieurs. 
Notre propos est de relier ces divergences aux différences de degré et de nature dans les investissements de forme que doivent consentir les deux institutions pour assurer l'employabilité de leurs diplômés, mais l'originalité de cette perspective et cette mise en corrélation mériteraient d'être validées, comme y invite l'approche ancrée de Glaser \& Strauss (1967), par des études de cas réalisées dans d'autres écoles.

La figure du manager s'appuie davantage sur des compétences relationnelles que techniques et les aptitudes managériales s'acquièrent surtout en situation réelle de travail et dans un contexte local. De ce fait, l'école de commerce doit investir plus fortement dans une formation "totale ", qui fait feu de tout bois, unifiant toutes les dimensions - scolaires, périscolaires, professionnelles - via un projet fil rouge, à toutes les formes d'expériences traduisibles en compétences. Cette mise en forme s'étend hors de la formation proprement dite, de l'espace géographique et de la temporalité de l'école et couvre toutes les dimensions de la vie de l'individu. Ce faisant, elle lui permet de se définir, en analogie au monde de l'entreprise, comme orienté "compétence " (et non savoirs scolaires), "projet " (c'est-à-dire tendu vers un but et optimisant le temps) et entreprise - ou marketing - de soi.

Quant à l'école d'ingénieurs, elle peut se permettre de ne s'intéresser qu'aux dimensions scolaire et intellectuelle de ses étudiants - et de proposer une professionnalisation "scindée ", où les compétences de natures différentes s'acquièrent dans des lieux différents, voire sont " déléguées " sans contrôle de l'école a priori ou a posteriori, aux entreprises, voire aux étudiants eux-mêmes. En effet, la figure de l'ingénieur est plus nette que celle du manager et la forme d'État fournit une équivalence plus générale et plus forte que la forme intermédiaire qu'offre l'école de commerce. Cette dernière se doit donc, pour " compenser » cette faiblesse, d'assurer, par la cohérence et l'omniprésence du "projet ", une équivalence entre compétences et salaire/emploi obtenus par les diplômés, que le titre d'ingénieur semble encore garantir.

Les écoles analysées dans cet article ne sont pas représentatives du paysage français des grandes écoles de commerce et d'ingénieurs. En effet, les négociations entre les injonctions extérieures, les contraintes environnementales et les aspirations identitaires sont propres à chaque établissement d'enseignement supérieur (Lemaître, 2009). Les mises en adéquation entre formation et emploi décrites ici demeurent donc contingentes aux écoles étudiées.

Nous voyons néanmoins dans la mobilisation par le projet, non seulement le mode de régulation "pivot» du néo-management (Boltanski \& Chiapello, 1999), mais aussi un mécanisme compensant les contours flous de la figure du manager, dont se passe celle de l'ingénieur, protégée - quand bien même elle tend à s'affaiblir - par une forme d'État. Face à la nécessité de réduire l'incertitude des employeurs et le champ des possibles, seule la " mise en forme " projet possède l'atout d'être reconnaissable et valorisable. Dès lors, elle conquiert progressivement l'espace-temps des étudiants, phagocytant le présent, excluant les inactifs et marginalisant les "sans-projet ». De son côté, le titre 
d'ingénieur, protégé par la robustesse de la forme d'État, continue à déployer (jusqu'à quand ?) un discours pédagogique plus vertical, plaçant le savoir académique au cœur des dispositifs de formation.

\section{Bibliographie}

Atlan D., Grelon A. (2009), "L'identité d'ingénieur chahutée par les évolutions du capitalisme ", Séminaire des Amis de l'École de Paris, compte-rendu de la séance du 25 mai.

Béduwé C., Mora V. (2016), "Comment se professionnalisent les étudiants ? ", in Lemistre P., Béduwé C. (dir.), Professionnalisation des publics et des parcours à l'université, Céreq échanges, $\mathrm{n}^{\circ} 3$, pp. 59-78.

Bernstein B. (2007), Pédagogie, contrôle symbolique et identité : théorie, recherche, critique, Québec, Les Presses de l'Université Laval.

Blanchard M. (2015), Les écoles supérieures de commerce. Sociohistoire d'une entreprise éducative en France, Paris, Classiques Garnier, coll. " Histoire des techniques ".

Boltanski L., Thévenot L. (1991), De la justification. Les économies de la grandeur, Paris, Gallimard, coll. «NRF Essais ».

Boltanski L., Chiapello E. (1999), Le nouvel esprit du capitalisme, Paris, Gallimard, coll. «NRF Essais ».

Bouffartigue P., Gadéa C. (1996), «Un héritage à l'épreuve. Bref panorama des évolutions dans la formation et l'emploi des ingénieurs en France ", Formation Emploi, n 53 , pp. 5-13.

Bourdieu P. (1989), La noblesse d'État. Grandes écoles et esprit de corps, Paris, Éditions de Minuit, coll. "Le sens commun ».

Bousquet N., Grandgérard C. (1996), « Du modèle des grandes écoles aux formations en partenariat. Quelles logiques de modernisation?", Formation Emploi, n 53, pp. 75-84.

Boutinet J.-P. (1990), Anthropologie du projet, Paris, Presses Universitaires de France, coll. "Psychologie d'aujourd'hui ".

Burchell G. (1993), "Liberal Government and Techniques of the Self", Economy and Society, Vol. 22, n 3, pp. 267-282.

Charles N. (2014), "Quand la formation ne suffit pas : la préparation des étudiants à l'emploi en Angleterre, en France et en Suède ", Sociologie du travail, vol. 56, $\mathrm{n}^{\circ} 3$, pp. 320-341.

Charles N. (2015), Enseignement supérieur et justice sociale. Sociologie des expériences étudiantes en Europe, Paris, La Documentation française, coll. " Etudes \& Recherche ». 
Eisenhardt M. (1989), "Building Theories from Case Study Research", The Academy of Management Review, 14 (4), October, pp. 532-550.

Finot A. (2000), Développer l'employabilité, Paris, Insep Consulting éditions.

Fournas (de) P. \& al. (2008), "Les grandes écoles, système dépassé ou produit d'avenir ? ", Le journal de l'école de Paris du management, $\mathrm{n}^{\circ} 72$, juillet-août, pp. 30-37.

Fournier G., Monette M., dir. (2000), Linsertion socioprofessionnelle : un jeu de stratégie ou un jeu de hasard?, Sainte-Foy, Les Presses de l'Université Laval.

Gautié J. (2003), Quelle Troisième Voie? Repenser l'articulation entre marché du travail et protection sociale, Paris, CEE, Document de travail $n^{\circ} 30$.

Gendron B. (2006), «Une analyse par l'économie des conventions des formations complémentaires d'initiative locale ", Cahiers de la maison des sciences économiques, n 2006.77.

Glaser B. \& Strauss A. (1967), The discovery of grounded theory: strategies for qualitative research, Chicago: Aldine de Gryter.

Gordon C. (1991), "Governmental rationality: an introduction", in Burchell G., Gordon C., Miller P. (eds), The Foucault Effect. Studies in Governmentality, Chicago, The University of Chicago Press.

Hatchuel A. (2006), « La naissance de l'ingénieur généraliste. L'exemple de l'Ecole des Mines de Paris ", Réalités industrielles, novembre, pp. 13-24.

Lahire B. (1996), "Les manières d'étudier ", Les Cahiers de l'OVE, n 2, Observatoire national de la Vie Étudiante, Paris, La Documentation française.

Lazuech G. (1999), L'exception française. Le modèle des grandes écoles à l'épreuve de la mondialisation, Rennes, Presses Universitaires de Rennes, coll. « Le Sens social ».

Lazuech G. (2000), « Recruter, être recrutable. L'insertion professionnelle des jeunes diplômés d'écoles d'ingénieurs et de commerce ", Formation Emploi, n 69, pp. 5-19.

Lemaitre D. (2011), " Professionnalisation et modèles professionnels dans les grandes écoles françaises ", Recherche \& Formation, n 66, pp. 93-106.

Lemaître D. (2009), "Le curriculum des grandes écoles en France : un modèle d'analyse inspiré de Basil Bernstein ", Revue française de pédagogie, n 166, janvier-mars, pp. 7-26.

Mouchot C. (2003), Méthodologie économique, Paris, Seuil, Mass Market Paperback.

Passeron J.-C., Revel J. (2005), " Penser par cas. Raisonner à partir de singularités ", in Passeron J.-C., Revel J., dir., Penser par cas, Paris, Editions EHESS, coll. "Enquête ", pp. 9-44. 
Périlleux T. (2005), «Se rendre désirable. L’employabilité dans l'État social actif et l'idéologie managériale ", in Vielle P., Pochet P. Cassiers I. (dir.), L'Etat social actif : vers un changement de pardigme, Berne, Peter Lang, pp. 301-322.

Rose N. (1992), "Governing the Enterprising Self”, in Heelas P., Morris P. (Eds.), The Values of the Enterprise Culture. The Moral Debate, London, Routledge, pp. 141-163.

Shinn T. (1978), « Des corps de l'État au secteur industriel : genèse de la profession d'ingénieur, 1750-1920 », Revue française de sociologie, vol. 19-1, pp. 39-71.

Sonntag M. (2007), « Les formations d'ingénieurs. Des formations professionnelles et professionnalisantes. Orientations, contenus, contextes ", Recherche \& Formation, n ${ }^{\circ} 55$, pp. 11-26.

Spence M. (1973), "Job Market Signaling", The Quarterly Journal of Economics, Vol. 87, $\mathrm{n}^{\circ}$ 3, August, pp. 355-374.

Thévenot L. (1986), "Les investissements de forme ", in Thévenot L., ed., Conventions économiques, Paris, Presses Universitaires de France, pp. 21-71.

Thomas D. R. (2006), "A General Inductive Approach for Analyzing Qualitative Evaluation Data", American Journal of Evaluation, 27(2), pp. 237-246. 